10.3

\title{
Высокочастотный резонансный магнитоэлектрический эффект в структуре FeCoSiB-AIN на диэлектрической подложке
}

\author{
(C) Д.А. Бурдин ${ }^{1}$, P. Hayes $^{2}$, Н.А. Экономов ${ }^{1}$, Д.В. Чашин ${ }^{1}$, Ю.К. Фетисов ${ }^{1}$ \\ ${ }^{1}$ МИРЭА - Российский технологический университет, Москва, Россия \\ 2 Institute for Materials Science, Christian-Albrechts-Universität zu Kiel, Kiel, Germany \\ E-mail: phantastic@mail.ru
}

Поступило в Редакцию 15 октября 2020 г.

В окончательной редакции 24 ноября 2020 г.

Принято к публикации 24 ноября 2020 г.

\begin{abstract}
Обнаружен высокочастотный магнитоэлектрический эффект в планарной структуре, содержащей ферромагнитный слой из $\mathrm{FeCoSiB}$ и пьезоэлектрический слой из $\mathrm{AlN}$, изготовленные методом магнетронного напыления на подложке из боросиликатного стекла. Структуру возбуждали магнитным полем на частоте толщинной акустической моды колебаний $32.4 \mathrm{MHz}$ и регистрировали генерируемое пьезослоем электрическое напряжение. Коэффициент магнитоэлектрического преобразования на частоте второй толщинной моды составляет $\alpha_{E} \approx 6 \mathrm{~V} / \mathrm{A}$ при постоянном магнитном поле смещения $120 \mathrm{~A} / \mathrm{m}$, соответствующем максимуму пьезомагнитного коэффициента ферромагнитного слоя.
\end{abstract}

Ключевые слова: высокочастотный магнитоэлектрический эффект, магнитострикция, пьезоэффект, композитная структура.

DOI: 10.21883/PJTF.2021.05.50675.18586

Магнитоэлектрические (МЭ) эффекты, т.е. взаимное преобразование электрических и магнитных полей в мультиферроидных материалах, широко исследуются для создания различных устройств информационной техники и микроэлектроники, таких как датчики магнитных полей, устройства обработки радиосигналов, элементы магнитной памяти, переключаемые электрическим полем [1]. В композитных гетероструктурах, содержащих ферромагнитные (ФМ) и пьезоэлектрические (ПЭ) слои, МЭ-эффекты возникают в результате комбинации магнитострикции ФМ-слоя и пьезоэлектричества в ПЭслое посредством передачи деформации через границу раздела. При этом на частотах, совпадающих с частотами акустических резонансов структуры, эффективность преобразования полей возрастает на два-три порядка из-за резкого увеличения деформаций [2].

МЭ-эффекты наблюдались в основном либо в диапазоне низких частот $\sim 10^{2}-10^{5} \mathrm{~Hz}$ при возбуждении изгибных или планарных акустических колебаний структур [3], либо в диапазоне частот $\sim 1-10 \mathrm{GHz}$ при возбуждении ферромагнитного резонанса в ФМ-слое структуры [4]. МЭ-эффекты в промежуточном высокочастотном диапазоне $0.3-30 \mathrm{MHz}$ остаются до сих пор практически неисследованными. Опубликовано всего несколько работ, где теоретически исследовались характеристики высокочастотных МЭ-эффектов в композитных структурах [5] и наблюдались резонансные эффекты в планарной структуре железоиттриевый гранат-лангатат на частоте $4.2 \mathrm{MHz}$ [6] и в структуре нитрид алюминия-аморфный ферромагнетик на частоте $60.5 \mathrm{MHz}$ [7]. Возможности исследования высокочастотных МЭ-эффектов осложнены падением эффективности
МЭ-преобразования с уменьшением размеров композитных резонаторов [8] на планарных акустических колебаниях и сложностями изготовления резонаторов малых размеров.

В настоящей работе обнаружен и исследован резонансный МЭ-эффект на толщинной моде акустических колебаний в пленочной структуре $\mathrm{FeCoSiB}-\mathrm{AlN}$, изготовленной на диэлектрической подложке. Применение толщинной моды колебаний вместо планарной позволило использовать пленочную структуру достаточно больших размеров в плоскости. Кратко описаны исследуемая структура, методики измерений и характеристики высокочастотного МЭ-эффекта.

Использованная структура схематически изображена на рис. 1. Основой структуры является пластина из боросиликатного стекла длиной $23 \mathrm{~mm}$, шириной $3 \mathrm{~mm}$ и толщиной $a=150 \mu \mathrm{m}$. На верхней стороне пластины методом магнетронного напыления изготовлен слой из пьезоэлектрического AlN толщиной $a_{p}=2 \mu \mathrm{m}$, a на нижней стороне - слой из аморфного ферромагнетика $\mathrm{FeCoSiB}$ толщиной $a_{m}=2 \mu \mathrm{m}$ [9]. Роль электродов пьезоэлектрического слоя выполняют подслой из $\mathrm{Pt}$ толщиной $0.1 \mu \mathrm{m}$ и верхний слой из $\mathrm{Au} / \mathrm{Cr}$ толщиной $0.1 \mu \mathrm{m}$. Пластина закреплена на одном конце на массивном основании, так что длина свободной части составляет $L=20 \mathrm{~mm}$. Структура помещена в постоянное магнитное поле смещения $H=0-1.6 \mathrm{kA} / \mathrm{m}$, созданное катушками Гельмгольца и направленное вдоль ее длинной оси. Возбуждающее переменное магнитное поле $h \cos (2 \pi f t)$ с частотой $f=1 \mathrm{kHz}-40 \mathrm{MHz}$ и амплитудой до $h=80 \mathrm{~A} / \mathrm{m}$, направленное параллельно $H$, создавалось с помощью низкоиндуктивной катушки с попе- 


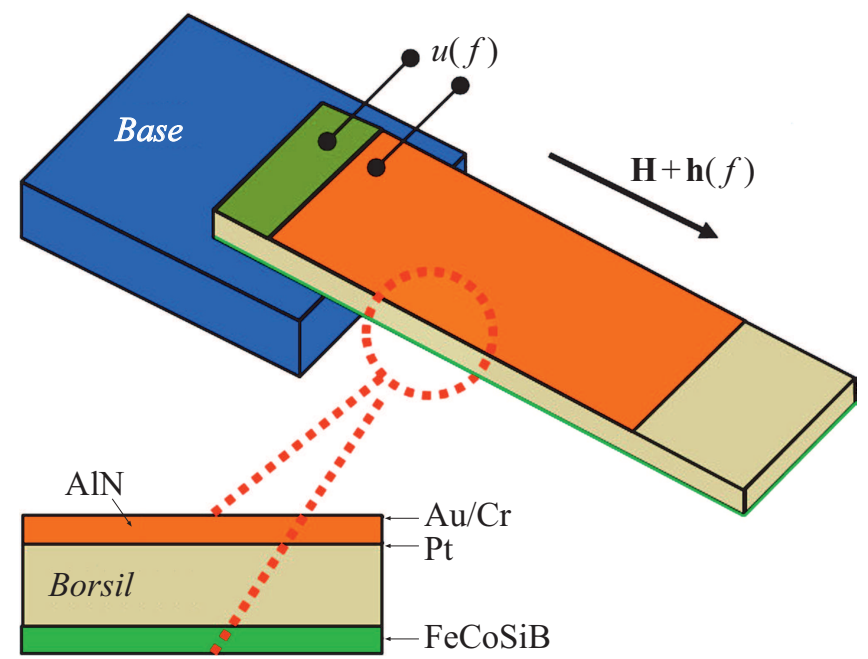

Рис. 1. Схематическое изображение структуры $\mathrm{FeCoSiB}-\mathrm{AlN}$.

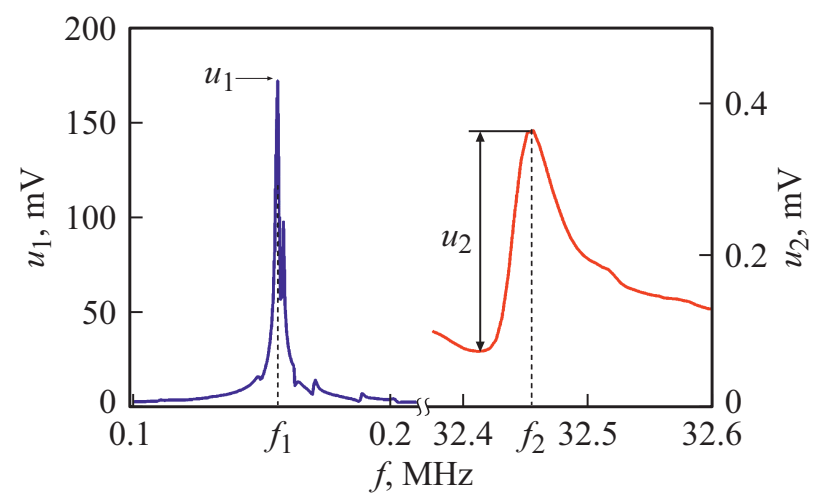

Рис. 2. Зависимость МЭ-напряжения $u$ от частоты $f$ возбуждающего магнитного поля при $H=120 \mathrm{~A} / \mathrm{m}$.

речными размерами $5 \times 9 \mathrm{~mm}$, длиной $22 \mathrm{~mm}$ и числом витков $N=80$, охватывающей свободную часть структуры. Активное сопротивление и индуктивность катушки равнялись соответственно $R=1.92 \Omega$ и $L=14.27 \mu \mathrm{H}$. МЭ-эффект регистрировался с помощью панорамного измерителя цепей Agilent 5061B. Для этого катушка подключалась к передающему порту измерителя цепей, а напряжение $u$ c электродов AlN-слоя подавалось на приемный порт измерителя. Регистрировались частотные зависимости коэффициента передачи $S_{21}$ структуры при возбуждающем напряжении менее $1 \mathrm{~V}$ и различных значениях поля $H$ в диапазоне частот $1 \mathrm{kHz}-40 \mathrm{MHz}$. Затем по коэффициенту передачи находилась амплитуда $u$ генерируемого МЭ-напряжения. Постоянное поле измерялось тесламетром LakeShore 421 с точностью $0.1 \mathrm{~A} / \mathrm{m}$. Полевая зависимость $\mu(H)$ магнитной проницаемости структуры определялась по полевой зависимости индуктивности катушки с помощью $R L C$-измерителя AKTAKОМ-3026 на частоте $10 \mathrm{kHz}$.

На рис. 2 показана зависимость амплитуды генерируемого структурой МЭ-напряжения $u$ от частоты $f$ возбуждающего магнитного поля при постоянном поле смещения $H=120 \mathrm{~A} / \mathrm{m}$. В области низких частот $0.1-0.2 \mathrm{MHz}$ на зависимости виден пик с частотой $f_{1} \approx 155.7 \mathrm{kHz}$, высотой $u_{1} \approx 173 \mathrm{mV}$ и добротностью $Q_{1} \approx 1300$, соответствующий возбуждению планарных колебаний структуры. В области высоких частот $32.4-32.6 \mathrm{MHz}$ на фоне сигнала прямой электромагнитной наводки виден второй пик с центральной частотой $f_{2} \approx 32.45 \mathrm{MHz}$, высотой $u_{2} \approx 0.25 \mathrm{mV}$ и добротностью $Q_{2} \approx 800$.

Для идентификации пиков напряжения на рис. 2 оценим частоты акустических резонансов исследованной структуры. Используем известные формулы для частот низших мод планарных и толщинных колебаний закрепленной на одном конце балки

$$
f_{1}=\left(k_{1} / 2 L\right) \sqrt{Y / \rho}, \quad f_{2}=\left(k_{2} / 2 a\right) \sqrt{Y / \rho},
$$

где коэффициенты могут принимать значения $k_{1}=1,3$, 5 и $k_{2}=1,2$. Толщины ФМ- и ПЭ-слоев учитывать не будем, поскольку они много меньше толщины диэлектрической подложки и слабо влияют на частоты колебаний. Подставляя в выражения (1) значения модуля Юнга и плотности боросиликатного стекла $Y \approx 7 \cdot 10^{10} \mathrm{~N} / \mathrm{m}^{2}$, $\rho \approx 2.23 \cdot 10^{3} \mathrm{~kg} / \mathrm{m}^{3}$, длину и толщину подложки, получаем частоты $f_{1} \approx 140 \mathrm{kHz}$ и $f_{2} \approx 36 \mathrm{MHz}$. Из сравнения измеренных и рассчитанных частот следует, что низкочастотный пик на рис. 2 соответствует возбуждению низшей моды планарных колебаний по длине структуры $\left(k_{1}=1\right)$, а высокочастотный пик - возбуждению второй моды $\left(k_{2}=2\right)$ колебаний по толщине структуры.

МЭ-коэффициенты для обоих пиков оценим по формуле $\alpha_{E}=e / h=u /\left(h a_{p}\right)$, где $e-$ амплитуда электрического поля, генерируемого структурой под действием магнитного поля $h$. Поле $h$ находили по току, протекающему через катушку. Для этого последовательно с катушкой включили безвыводное сопротивление величиной $R_{0}=1 \Omega$ с индуктивностью $L_{0}=27 \mathrm{nH}$ и измерили амплитуду напряжения $V$ на сопротивлении на частотах обоих пиков. Рассчитав амплитуду тока как $I=V / \sqrt{R_{0}^{2}+\left(2 \pi f L_{0}\right)^{2}}$, по формуле для поля длинного соленоида $h=I N$, где $N$ - число витков на единицу длины, находим амплитуды полей: $h_{1} \approx 73 \mathrm{~A} / \mathrm{m}$ на частоте $155.7 \mathrm{kHz}$ и $h_{2} \approx 20 \mathrm{~A} / \mathrm{m}$ на частоте $32.4 \mathrm{MHz}$. Окончательная оценка с использованием данных рис. 2 дает МЭ-коэффициент $\alpha_{E 1} \approx 1200 \mathrm{~V} / \mathrm{A}$ на частоте $f_{1}$ планарных колебаний структуры и $\alpha_{E 2} \approx 6 \mathrm{~V} / \mathrm{A}$ на частоте $f_{2}$ толщинных колебаний структуры.

Для подтверждения МЭ-природы высокочастотного пика была измерена зависимость напряжения $u_{2}$ от постоянного поля $H$, показанная на рис. 3. Видно, что $u_{2}$ сначала примерно линейно растет с увеличением $H$, достигает максимума в поле $H_{m} \approx 120 \mathrm{~A} / \mathrm{m}$, а затем асимптотически стремится к нулю по мере насыщения ФМ-слоя структуры. Именно такая полевая зависимость напряжения характерна для линейных МЭ-эффектов в планарных структурах [3]. Напряжение максимально в 


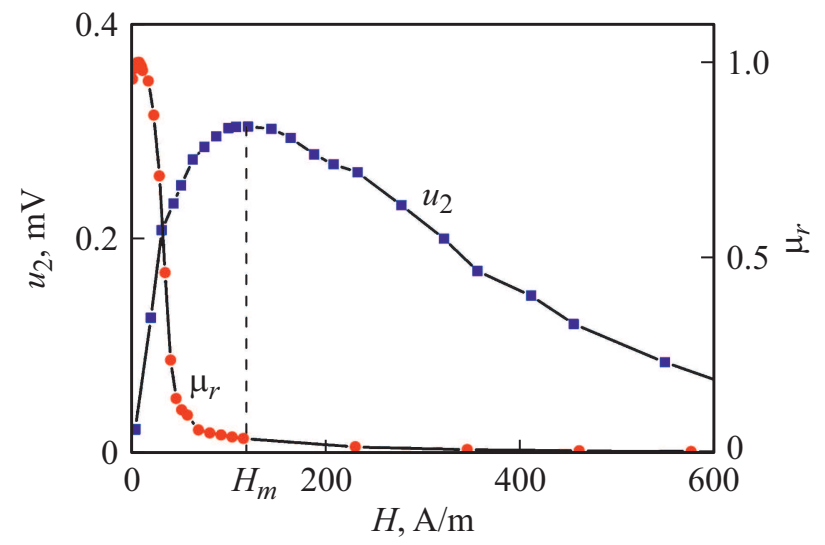

Рис. 3. Зависимость МЭ-напряжения $u_{2}$ и нормированной магнитной проницаемости $\mu_{r}$ от постоянного магнитного поля $H$.

поле $H_{m}$, соответствующем максимуму пьезомагнитного коэффициента $q(H)=\partial \lambda / \partial H$ ФМ-слоя структуры, где $\lambda(H)$ - полевая зависимость магнитострикции. Чтобы убедиться, что изменение магнитной проницаемости $\mu$ при увеличении $H$ не влияет на величину $u_{2}$, была измерена полевая зависимость нормированной магнитной проницаемости $\mu_{r}(H)=\mu(H) / \mu_{\max }$ ФМ-слоя структуры ( $\mu_{\max }$ - максимальная величина магнитной проницаемости), приведенная на том же рис. 3. Видно, что проницаемость максимальна в полях $H=0-20 \mathrm{~A} / \mathrm{m}$, а затем резко падает уже в поле $H \approx 80 \mathrm{~A} / \mathrm{m}$. Значительное отличие характерных полей максимума для $u_{2}(H)$ и $\mu_{r}(H)$ еще раз подтверждает МЭ-природу высокочастотного пика.

В заключение отметим, что акустические колебания по толщине структуры возбуждаются из-за изменения толщины ФМ-слоя, обусловленного магнитострикционной деформацией слоя в плоскости. Коэффициент Пуассона, определяющий связь деформации ФМ-слоя в плоскости и изменение его толщины, для $\mathrm{FeCoSiB}$ составляет $\gamma \approx 0.3$, что приводит к соответствующему уменьшению МЭ-коэффициента для высокочастотного эффекта. Уменьшению МЭ-коэффициента способствует также демпфирующее воздействие подложки [10]. Можно надеяться, что выбор оптимальной толщины слоев композитной структуры позволит повысить эффективность преобразования полей при высокочастотном МЭ-эффекте.

\section{Финансирование работы}

Работа поддержана Российским фондом фундаментальных исследований (грант 20-07-00811).

\section{Конфликт интересов}

Авторы заявляют, что у них нет конфликта интересов.

\section{Список литературы}

[1] C. Tu, Z. Chu, B. Spetzler, P. Hayes, C.-Z. Dong, X.-F. Liang, H.-H. Chen, Y.-Y. He, Y.-Y. Wei, H. Lin, Y.-H. Lin, J. McCord, F. Faupel, E. Quandt, N.-X. Sun, Materials, 12, 2259 (2019). doi.org/10.3390/ma12142259.

[2] M.I. Bichurin, D.A. Filippov, V.M. Petrov, V.M. Laletsin, N. Paddubnaya, G. Srinivasan, Phys. Rev. B, 68, 132408 (2003). doi.org/10.1103/PhysRevB.68.132408

[3] C.-W. Nan, M.I. Bichurin, S. Dong, D. Viehland, G. Srinivasan, J. Appl. Phys., 103, 031101 (2008). doi.org/10.1063/1.2836410

[4] G. Srinivasan, Y.K. Fetisov, Ferroelectrics, 342, 65 (2006). doi.org/10.1080/00150190600946195

[5] V.M. Petrov, A.F. Saplev, O.I. Matushev, J. Phys.: Conf. Ser., 1352, 012037 (2019).

[6] M.I. Bichurin, R.V. Petrov, V.M. Petrov, Appl. Phys. Lett., 103, 092902 (2013). doi.org/10.1063/1.4819477

[7] T. Nan, H. Lin, Y. Gao, A. Matyushov, G. Yu, H. Chen, N. Sun, S. Wei, Z. Wang, M. Li, X. Wang, A. Belkessam, R. Guo, B. Chen, J. Zhou, Z. Qian, Y. Hui, M. Rinaldi, M.E. McConey, B.M. Howe, Z. Hu, J.G. Jones, G.J. Brown, N.X. Sun, Nature Commun., 8, 296 (2017). doi.org/10.1038/s41467-017-00343-8

[8] F.A. Fedulov, D.V. Chashin, L.Y. Fetisov, A.M. Kharlamova, Y.K. Fetisov, IEEE Sensors Lett., 4, 2501104 (2020). DOI: $10.1109 /$ LSENS.2020.3021757

[9] P. Hayes, V. Schell, S. Salzer, D. Burdin, E. Yarar, A. Piorra, R. Knochel, Y.K. Fetisov, E. Quandt, J. Phys. D: Appl. Phys., 51, 354002 (2018). doi.org/10.1088/1361-6463/aad456

[10] Д.А. Филиппов, Т.О. Фирсова, Вестн. Новгород. ун-та, 55, 47 (2010). 